\title{
A New Modelling Approach of Evaluating Preventive and Reactive Strategies for Mitigating Supply Chain Risks
}

\author{
Abroon Qazi ${ }^{1, *}, J^{*}$ hn Quigley ${ }^{1}$, Alex Dickson ${ }^{1}$, and Barbara Gaudenzi ${ }^{2}$ \\ ${ }^{1}$ Strathclyde Business School, University of Strathclyde, Glasgow, United Kingdom \\ \{abroon.qazi, j.quigley, alex.dickson\}@strath.ac.uk \\ ${ }^{2}$ Faculty of Business Economics, University of Verona, Verona, Italy \\ barbara.gaudenzi@univr.it
}

\begin{abstract}
Supply chains are becoming more complex and vulnerable due to globalization and interdependency between different risks. Existing studies have focused on identifying different preventive and reactive strategies for mitigating supply chain risks and advocating the need for adopting specific strategy under a particular situation. However, current research has not addressed the issue of evaluating an optimal mix of preventive and reactive strategies taking into account their relative costs and benefits within the supply network setting of interconnected firms and organizations. We propose a new modelling approach of evaluating different combinations of such strategies using Bayesian belief networks. This technique helps in determining an optimal solution on the basis of maximum improvement in the network expected loss. We have demonstrated our approach through a simulation study and discussed practical and managerial implications.
\end{abstract}

Keywords: Supply chain risks; Preventive and reactive strategies; Bayesian belief networks; Network expected loss; Simulation study

\section{$1 \quad$ Introduction}

Supply chains have become complex because of the globalization and outsourcing in manufacturing industries. Supply chain risk is characterized by both the probability of an event and its severity given that an event occurs. Supply chain risk management (SCRM) is an active area of research that deals with the overall management of risk events ranging across the entire spectrum of the supply chain including external risk factors. "SCRM aims to identify the potential sources of supply chain risk and implement appropriate actions to avoid or contain supply chain vulnerability" [1]. Vulnerability is defined as an exposure to serious disturbances from risks within the supply chain as well as risks external to the supply chain [2]. Supply chain risk is an event that may cause disruption to the flow of activities within the supply chain. Recently, there has been a shift in the interest of researchers towards exploring impact of disruption on global supply chains. Global sourcing and lean operations are the main drivers of supply chain disruptions [3]. 
Bayesian belief network (BBN) is a an acyclic directed graphical model comprising nodes representing uncertain variables and arcs indicating causal relationships between variables whereas the strength of dependency is represented by the conditional probability values. BBNs have started gaining the interest of researchers in modelling supply chain risks [4]. BBNs offer a unique feature of modelling risks combining both the statistical data and subjective judgment in case of non-availability of data [5]. Researchers have used the BBNs to model specific domains of supply chain risks and validated these models through case studies.

\subsection{Research Problem and Contribution}

It is extremely important to consider the interdependency between risks in modelling supply chain risks. However, capturing the probabilistic interaction between risks and resulting losses is not sufficient for managing risks as risk management process necessitates selecting cost-effective strategies. Selection of optimal mix of risk mitigation strategies has never been explored within the realm of interconnected risks across different segments of a supply network. This paper bridges the research gap and presents a new modelling approach of evaluating mix of preventive and reactive strategies taking into account the supply network configuration, interdependency between risks and associated costs and benefits of different combinations of risk mitigation strategies. The technique will help researchers develop robust models of managing supply chain risks and benefit practitioners in understanding interaction between risks and selecting optimal mix of risk mitigation strategies.

\subsection{Outline}

We present brief overview of the research conducted in SCRM in Section 2. New modelling approach of evaluating risk mitigation strategies is described in Section 3. Application of the proposed method is demonstrated through a simulation study in Section 4. Furthermore, results are also discussed in detail followed by the explication of managerial implications. Finally, conclusion and future research agenda are presented in Section 5.

\section{Literature Review}

\subsection{Supply Chain Risk Management}

Risk management is an established field in some areas of organizational life like finance but it is still a developing theme within the realm of Supply chain management [6]. Despite the ongoing debate on the objective and subjective nature of risk, there is a consensus among researchers on treating the risk management as a process comprising three stages of risk identification, risk estimation and risk evaluation [7]. "SCRM is the management of supply chain risks through coordination or collaboration amongst the supply chain partners so as to ensure profitability and continuity" [8]. 
Simulation has been extensively used by researchers in modeling supply chain risks. It provides a systematic approach for understanding the interactive impact of factors for different scenarios. Simulation techniques used in the realm of supply chain risk management include agent-based modeling, Monte Carlo simulation, discrete event simulation, system dynamics modeling and Petri-Net simulation [9]. Researchers have also used mixed methods in their research. Analytical hierarchy process has been considered as an effective technique for modelling and managing supply chain risks [10].

The major limitation of existing models is their lack of capturing the holistic nature of supply chain risks. Many techniques are not able to account for risk propagation [11]. Furthermore, existing methods and models have not taken into consideration the network configuration of a supply chain. The limited focus of these models in solving specific problems results in evaluating locally optimal solutions. BBNs present a useful technique of capturing interaction between risk events and performance measures [4]. Another advantage of using BBNs for modelling supply chain risks is the ability of back propagation that helps in determining the probability of an event that may not be observed directly. There are certain problems associated with the use of BBNs. Firstly, with the increase in number of nodes representing supply chain risks, a considerable amount of data is required in populating the network with (conditional) probability values and it might not be feasible to elicit huge data from the experts. Secondly, there are computational challenges associated with the increase in number of nodes.

\subsection{Bayesian Belief Network based Models}

Lockamy and McCormack [12] developed a model for benchmarking supplier risks incorporating risk events related to supplier network, internal operations and external factors. They used surveys and interviews for collection of data from both the internal and external company sources and applied the model on a group of 15 automotive casting suppliers for a major automotive company in US. Dogan and Aydin [13] developed a supplier selection model combining Total Cost of Ownership and BBN methods and applied the model in automotive industry to help Tier-1 suppliers select their own suppliers. They found the method to be suitable in dealing with incomplete or uncertain information of buyers about the suppliers.

Badurdeen et al. [4] developed supply chain risk taxonomy and a risk network map capturing interdependencies between risks and applied the model on the Boeing company and its Tier 1 Suppliers. Their model presents an effective tool to capture the interaction of risk factors and helps in identifying key suppliers. Risk propagation across multiple tiers is not explored in their study. Furthermore, modelling of resulting losses and mitigation strategies with associated costs is not considered and therefore, risk management process is not explored through BBNs comprehensively.

Garvey et al. [11] presented a Bayesian network approach of modelling risk propagation in a supply network. Their proposed model takes into consideration the interdependencies between risks and the structure of a supply network. They introduced different risk measures on the basis of this model and conducted a simulation study in 
order to demonstrate the use of risk measures in a supply network setting. However, evaluation of their proposed risk measures is not feasible in case of a complex network structure. Furthermore, they did not focus on the risk evaluation stage of risk management process.

\subsection{Limitations and Research Gap}

Most of the existing studies in SCRM have focused on specific domains in supply chain without considering the holistic view. Qualitative techniques are not able to capture the interaction of risks exclusively whereas many quantification methods treat risks as independent [4], [13]. Limited studies have considered modelling interdependency between risks and resulting losses. However, it is not sufficient to model the probabilistic interaction between risks and resulting losses. Risk evaluation is an equally important stage of the risk management process that necessitates evaluating the costs and benefits associated with different combinations of risk mitigation strategies. Risk evaluation has gained limited attention of the researchers in SCRM and no study has focused on integrating the probabilistic interaction between risks, resulting losses and impact of mitigation strategies. It is, therefore, important to investigate an effective approach of not only assessing risks but also evaluating different mitigation strategies within a framework of interconnected risks and mitigation strategies.

\section{New Modelling Approach}

Based on the efficacy of BBNs in capturing interdependencies between risks, we consider BBN based modeling of a supply network as an effective approach. Such a modeling technique can help managers visualize supply chain risks and take effective mitigation strategies [5], [9]. BBNs have been already explored in the literature of SCRM, however, our proposed BBN based modelling approach is unique in terms of introducing new risk measures that capture the impact of loss propagation across the entire network and demonstrating the efficacy of BBNs in evaluating risk mitigation strategies.

\subsection{Framework}

We follow the butterfly view of supply chain risks ranging from the causes to actual risk events to consequences [14]. Furthermore, we classify risks as process, upstream, downstream and external risks. Process risks relate to the risks directly associated with the main focal firm and comprise inventory, operational, quality and management risks. Downstream and upstream risks arise from the interaction between the focal firm and its customers and suppliers respectively. External risks are driven by external events like weather, earthquakes, political and market forces [15]. Supply chain risks can be considered as an interconnected web of events spanning across the entire network as shown in Fig. 1. 


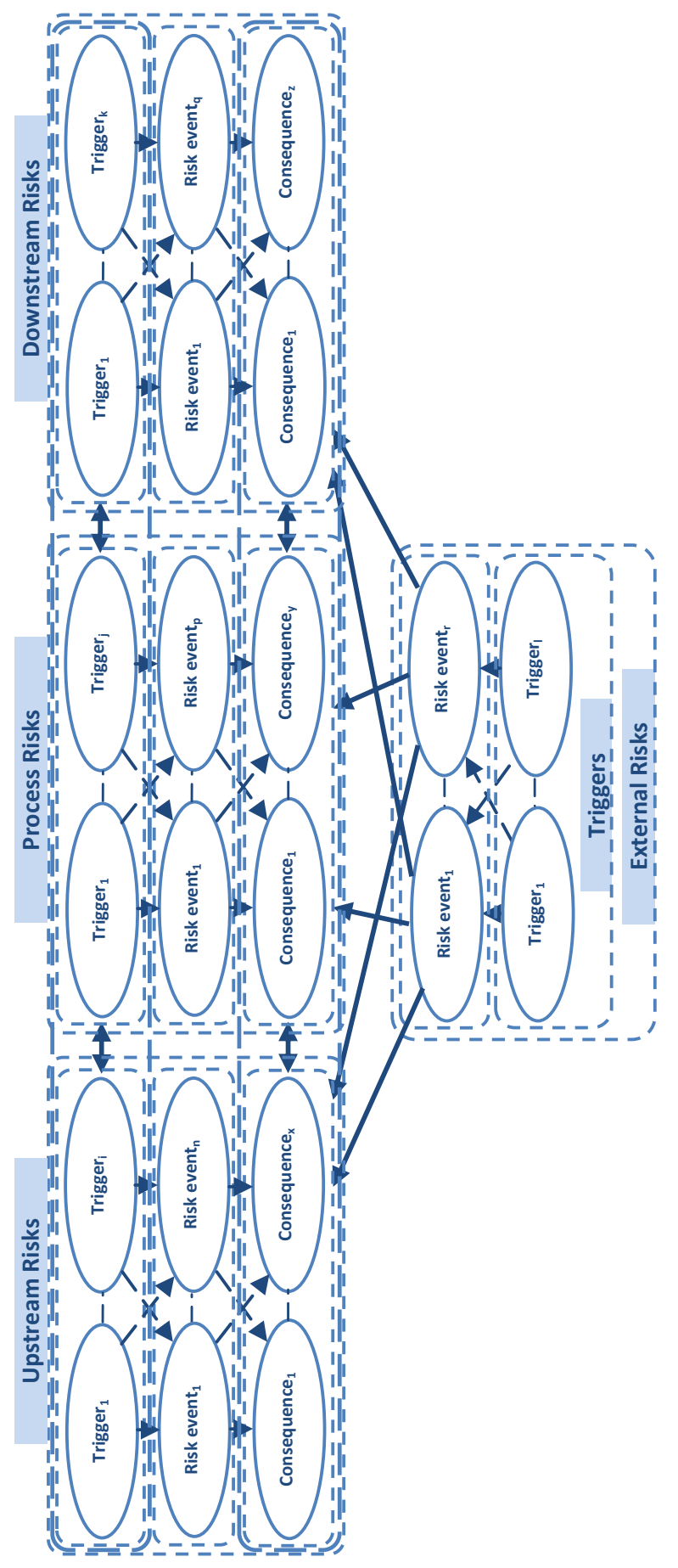

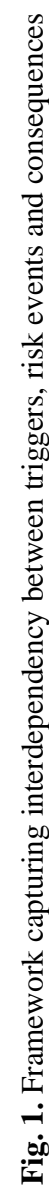




\subsection{Assumptions}

Our model is based on following assumptions:

1. Entire structure of the supply network is known

2. Risk triggers, events and consequences can be assigned to different locations and links between the locations and furthermore, all stakeholders agree to share such information

3. All random variables are represented by binary states

4. Conditional probability values and resulting losses can be elicited from the stakeholders and the resulting Bayesian network represents close approximation to the actual perceived risks and interdependency between different risks

5. Each mitigation strategy comprises three states including options of taking no action, adopting preventive strategy and implementing reactive strategy

6. Costs associated with the implementation of either strategy at important nodes are known

\subsection{Model and Risk Measures}

A discrete supply chain risk diagram $N=(X, G, P, L)$ is a four-tuple consisting of

- a directed acyclic graph $(D A G), G=(V, E)$, with nodes, $V$, representing discrete risk events, $X_{R}$, discrete risk mitigation strategies, $X_{S}$, and loss functions, $L$, and directed links, $E$, encoding dependence relations

- a set of conditional probability distributions, $P$, containing a distribution, $P\left(X_{R} \mid X_{p a(R)}\right)$, for each risk event, $X_{R}$

- a set of loss functions, $L$, containing one loss function, $l\left(X_{p a(V)}\right)$, for each node $v$ in the subset $V_{l} \in V$ of loss nodes.

$$
E L(X)=\prod_{X_{v} \in X_{R}} P\left(X_{v} \mid X_{p a(v)}\right) \sum_{w \in V_{L}} l\left(X_{p a(w)}\right)
$$

where $E L(X)$ is the expected loss across entire supply network

Definitions. Following terms relate to the combination of risk mitigation strategies corresponding to two different configurations of the supply network:

- Standard Configuration (SC). Supply network is considered to be in its standard configuration when risk mitigation strategies selected in the Bayesian network reflect real-time profile of these strategies in the supply network.

- Contingency Configuration (CC). Supply network is considered to be in its contingency configuration when the combination of risk mitigation strategies satisfies the objective function.

Risk Measures. We introduce two risk measures in order to evaluate the relative contribution of each risk factor towards the loss propagation across entire network. 
- Loss Propagation Containment Measure (LPCM). Loss propagation containment measure is the ratio between relative improvement in the network expected loss corresponding to complete mitigation of the risk factor and network expected loss for the standard configuration.

$$
\begin{gathered}
L P C M_{X_{R_{i}}}=\frac{E L(X)-E L\left(X \mid X_{R_{i}}=\text { false }\right)}{E L(X)_{S C}} \\
\text { Avg. } L P C M(\overline{L P C M})=1 / n \sum_{1}^{n} L P C M_{X_{R_{i}}}
\end{gathered}
$$

- Loss Propagation Spread Measure (LPSM). Loss propagation spread measure is the ratio between range of network expected loss corresponding to the two extreme states of the risk factor and network expected loss for the standard configuration.

$$
\begin{aligned}
& \operatorname{LPSM}_{X_{R_{i}}}=\frac{E L\left(X \mid X_{R_{i}}=\text { true }\right)-E L\left(X \mid X_{R_{i}}=\text { false }\right)}{E L(X)_{S C}} \\
& \text { Avg. LPSM }(\overline{L P S M})=1 / n \sum_{1}^{n} L_{P S M_{X_{R_{i}}}}
\end{aligned}
$$

\subsection{Modelling Process}

Following steps must be followed in developing the Bayesian network based model of a given supply network and evaluating the optimal combination of mitigation strategies:

1. Define the boundaries of supply network and identify stakeholders

2. Following the supply network process flow, classify risks as triggers, risk events and consequences on the basis of input received from each stakeholder

3. Refine the qualitative structure of the resulting network involving all stakeholders

4. Elicit (conditional) probability values, loss values resulting from risks and costs associated with implementing different mitigation strategies and populate the Bayesian network with all values

5. Define the objective function

6. Run the model and export array of values corresponding to different combinations of strategies to Microsoft Excel

7. Repeat the previous step for instantiation of each risk factor to the extreme states

8. Analyze the results and select optimal combination of strategies satisfying the objective function

9. Validate the model output involving stakeholders

\section{Simulation Study}

We demonstrate our proposed method through a simple supply network [11] as shown in Fig. 2. The model was developed in GeNIe software. The supply network comprises a raw material source, two manufacturers, a warehouse and retailer. Risks are represented by oval shaped nodes whereas resulting losses and control strategies are 
represented by diamond and rectangular shaped nodes respectively. Each risk factor is represented by a unique number appearing at top of the node. Though each domain of the supply network may comprise a number of triggers, risk events and consequences, we consider limited risks for the sake of simplicity. Furthermore, we consider the significance of losses and mitigation strategies at the interface of different domains. However, it is equally important to consider internal risks and related mitigation strategies in managing supply chain risks.

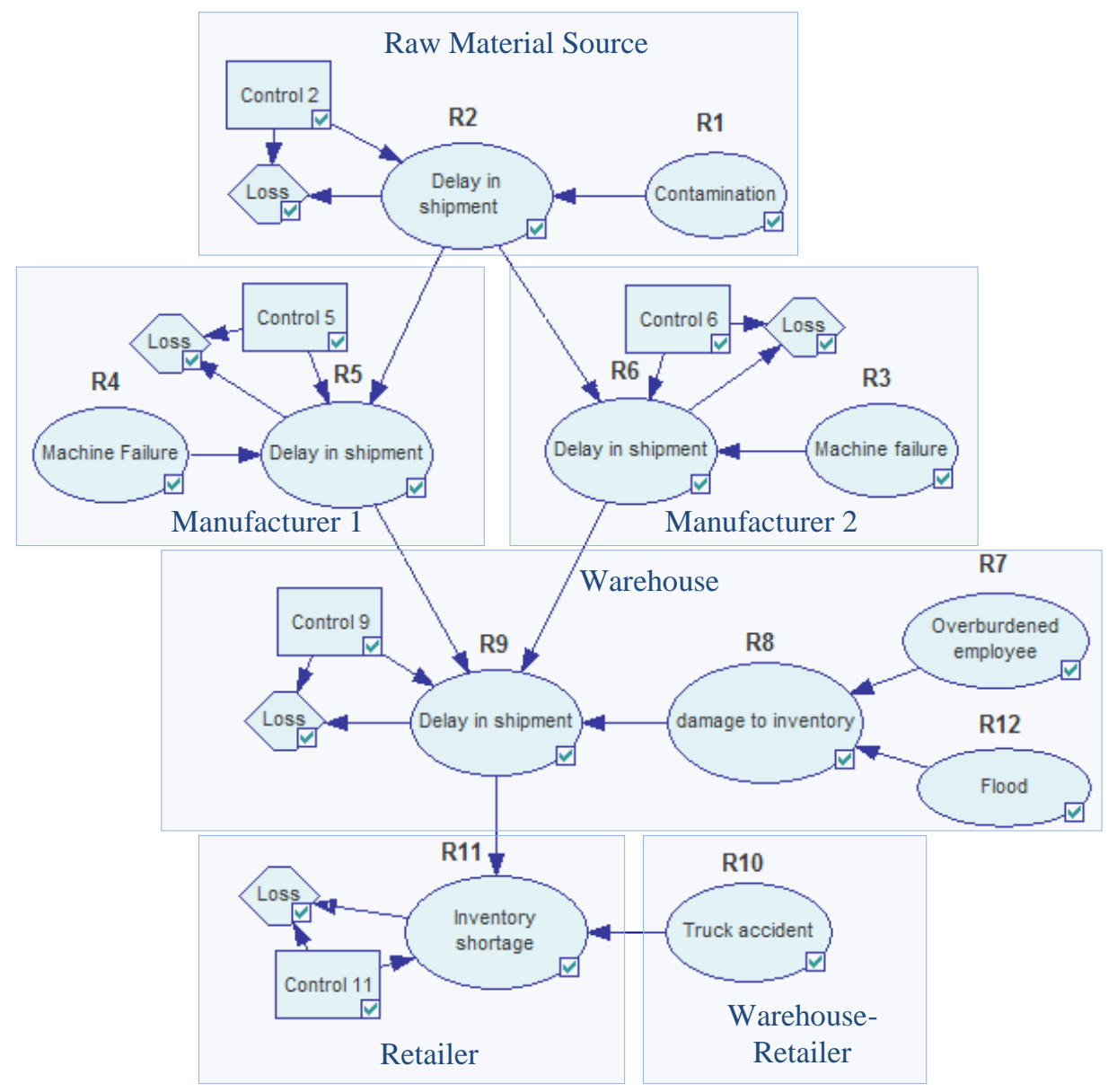

Fig. 2. Bayesian network based model of a supply network (adopted from Garvey et al. [11])

Each control node comprises three states; no mitigation strategy, preventive strategy and reactive strategy. (Conditional) probability values of risks (given no mitigation strategy) for the network are shown in Table 1. Loss values and costs associated with each strategy are shown in Table 2 . We also assume that under standard configuration, 'no mitigation strategy' is selected for all the control nodes. Conditional probability values of risks (given preventive or reactive strategy) are given as follows: 


$$
\begin{gathered}
P(\text { risk }=\text { True }(T) \mid \text { strategy }=\text { Preventive })=0.0001 \\
P(\text { risk }=\text { False }(F) \mid \text { strategy }=\text { Preventive })=0.9999 \\
P(\text { risk }=T \mid \text { strategy }=\text { Reactive })=P(\text { risk }=T \mid \text { strategy }=N o) \\
P(\text { risk }=F \mid \text { strategy }=\text { Reactive })=P(\text { risk }=F \mid \text { strategy }=N o)
\end{gathered}
$$

\begin{tabular}{|c|c|c|c|c|c|c|c|c|c|}
\hline \multirow{2}{*}{\multicolumn{4}{|c|}{ Parents }} & \multicolumn{6}{|c|}{$P($ risk|parents $)$} \\
\hline & & & & R1 & R2 & R3 & R4 & R5 & R6 \\
\hline R1 & R2 & R3 & $\mathrm{R} 4$ & $\mathrm{~T}$ & $\mathrm{~T}$ & $\mathrm{~T}$ & $\mathrm{~T}$ & $\mathrm{~T}$ & $\mathrm{~T}$ \\
\hline
\end{tabular}

Table 1. $($ Conditional $)$ probability values $(P($ risk $=F \mid$ parents $)=1-P($ risk $=$ T|parents $)$

$\mathrm{T}$

F

0.3

0.2

0.3

$\begin{array}{ll}0.7 & \\ 0.4 & \\ 0.6 & \\ 0.1 & \\ & 0.9 \\ & 0.6 \\ & 0.5 \\ & \\ & 0.2\end{array}$

\begin{tabular}{|c|c|c|c|c|c|c|c|c|c|c|c|c|}
\hline \multirow{2}{*}{\multicolumn{7}{|c|}{ Parents }} & \multicolumn{6}{|c|}{$P$ (risk|parents) } \\
\hline & & & & & & & R7 & $\mathrm{R} 8$ & R9 & R10 & R11 & R12 \\
\hline R5 & R6 & R7 & $\mathrm{R} 8$ & R9 & R10 & R12 & $\mathrm{T}$ & $\mathrm{T}$ & $\mathrm{T}$ & $\mathrm{T}$ & $\mathrm{T}$ & $\mathrm{T}$ \\
\hline & & & & & & & 0.4 & & & & & \\
\hline & & $\mathrm{T}$ & & & & $\mathrm{T}$ & & 0.8 & & & & \\
\hline & & $\mathrm{T}$ & & & & $\mathrm{F}$ & & 0.3 & & & & \\
\hline & & $\mathrm{F}$ & & & & $\mathrm{T}$ & & 0.6 & & & & \\
\hline & & $\mathrm{F}$ & & & & $\mathrm{F}$ & & 0.2 & & & & \\
\hline $\mathrm{T}$ & $\mathrm{T}$ & & $\mathrm{T}$ & & & & & & 0.9 & & & \\
\hline $\mathrm{T}$ & $\mathrm{T}$ & & $\mathrm{F}$ & & & & & & 0.5 & & & \\
\hline $\mathrm{T}$ & $\mathrm{F}$ & & $\mathrm{T}$ & & & & & & 0.6 & & & \\
\hline $\mathrm{T}$ & $\mathrm{F}$ & & $\mathrm{F}$ & & & & & & 0.3 & & & \\
\hline $\mathrm{F}$ & $\mathrm{T}$ & & $\mathrm{T}$ & & & & & & 0.4 & & & \\
\hline $\mathrm{F}$ & $\mathrm{T}$ & & $\mathrm{F}$ & & & & & & 0.3 & & & \\
\hline $\mathrm{F}$ & $\mathrm{F}$ & & $\mathrm{T}$ & & & & & & 0.3 & & & \\
\hline $\mathrm{F}$ & $\mathrm{F}$ & & $\mathrm{F}$ & & & & & & 0.2 & & & \\
\hline & & & & $\mathrm{T}$ & $\mathrm{T}$ & & & & & & 0.9 & \\
\hline & & & & $\mathrm{T}$ & $\mathrm{F}$ & & & & & & 0.7 & \\
\hline & & & & $\mathrm{F}$ & $\mathrm{T}$ & & & & & & 0.6 & \\
\hline & & & & $\mathrm{F}$ & $\mathrm{F}$ & & & & & & 0.2 & \\
\hline & & & & & & & & & & & & 0.2 \\
\hline
\end{tabular}


Table 2. Loss values and costs for different mitigation strategies

\begin{tabular}{cccc}
\hline Risk & $\begin{array}{c}\text { Loss (no } \\
\text { mitigation } \\
\text { strategy) }\end{array}$ & $\begin{array}{c}\text { Loss (preventive } \\
\text { strategy) [Cost] }\end{array}$ & $\begin{array}{c}\text { Loss (reactive } \\
\text { strategy) [Cost] }\end{array}$ \\
\hline R2 & 500 & $500[300]$ & $250[100]$ \\
R5 & 100 & $100[70]$ & $50[30]$ \\
R6 & 220 & $220[130]$ & $110[70]$ \\
R9 & 940 & $940[600]$ & $470[300]$ \\
R11 & 30 & $30[25]$ & $15[10]$ \\
\hline
\end{tabular}

\subsection{Problem Statement}

Given different options of preventive and reactive strategies and associated costs available at different nodes of the supply network, what is the optimal combination of these strategies yielding maximum improvement in the network expected loss taking into consideration the associated mitigation cost?

Objective Function. In this study, we aim to maximize the improvement in network expected loss keeping in view the costs associated with different mitigation strategies.

$$
\max _{\gamma_{x_{S}} \in \gamma_{X_{S}}} E L\left(X_{\gamma_{X_{S C}}}\right)-E L\left(X_{\gamma_{x_{S}}}\right)-C_{\gamma_{x_{S}}}
$$

where $\gamma_{X_{S C}}$ is the combination of different states of $n$ mitigation strategies under standard configuration

$\gamma_{X_{S}}$ is a set of all possible orderings of different states of $n$ mitigation strategies $\left(x_{s_{1}} \times x_{s_{2}} \times \ldots \times x_{s_{n}}\right)$

$C_{\gamma_{x_{S}}}$ is the cost of implementing $\gamma_{x_{S}}$ combination of mitigation strategies

\subsection{Results and Discussion}

Once the Bayesian network was updated, array of network expected loss values was exported to Microsoft Excel. Because of availability of three options at each of the five control nodes, there were 243 different combinations of control strategies. Under standard configuration with no mitigation strategy selected at any control node, the network expected loss was 747.52 units. Risk measures of all risk factors are shown in Table 3. Risk spectrum representing the graphical dimension of risk measures is shown in Fig. 3. R9 is the most important risk factor having maximum values of LPCM and LPSM. As risk factors appearing at the interface of different supply network domains were considered important in our model, and therefore assigned loss values and control strategies, high values of LPCM and LPSM could be observed for all these risk factors. If other risk factors were also assigned loss values, the resulting risk measures would be higher in magnitude. Furthermore, external risk triggers affecting multiple organizations within the network would also result in achieving high values of the risk measures. Without considering the cost factor, it seems viable to 
implement a control strategy for mitigating R9, however, it might not be feasible after capturing dynamics of all significant factors.

Table 3. Risk measures of risk factors under standard configuration

\begin{tabular}{|c|c|c|c|c|}
\hline \multirow[b]{2}{*}{ Risk } & \multicolumn{4}{|c|}{ Standard Configuration } \\
\hline & $\begin{array}{l}\text { Expected Loss } \\
\text { (True) }\end{array}$ & $\begin{array}{c}\text { Expected Loss } \\
\text { (False) }\end{array}$ & LPCM & LPSM \\
\hline $\mathrm{R} 1$ & 963.72 & 603.39 & 0.1928 & 0.4820 \\
\hline R2 & 1107.85 & 387.19 & 0.4820 & 0.9641 \\
\hline R3 & 834.42 & 725.80 & 0.0291 & 0.1453 \\
\hline R4 & 834.21 & 710.37 & 0.0497 & 0.1657 \\
\hline R5 & 1053.26 & 567.96 & 0.2402 & 0.6492 \\
\hline R6 & 1068.74 & 473.88 & 0.3661 & 0.7958 \\
\hline R7 & 760.79 & 738.68 & 0.0118 & 0.0296 \\
\hline $\mathrm{R} 8$ & 871.34 & 687.09 & 0.0808 & 0.2465 \\
\hline R9 & 1431.92 & 352.66 & 0.5282 & 1.4438 \\
\hline $\mathrm{R} 10$ & 753.40 & 743.60 & 0.0052 & 0.0131 \\
\hline R11 & 964.46 & 518.39 & 0.3065 & 0.5967 \\
\hline \multirow[t]{2}{*}{$\mathrm{R} 12$} & 812.38 & 731.31 & 0.0217 & 0.1085 \\
\hline & $E L(X)$ & 747.52 & & \\
\hline
\end{tabular}

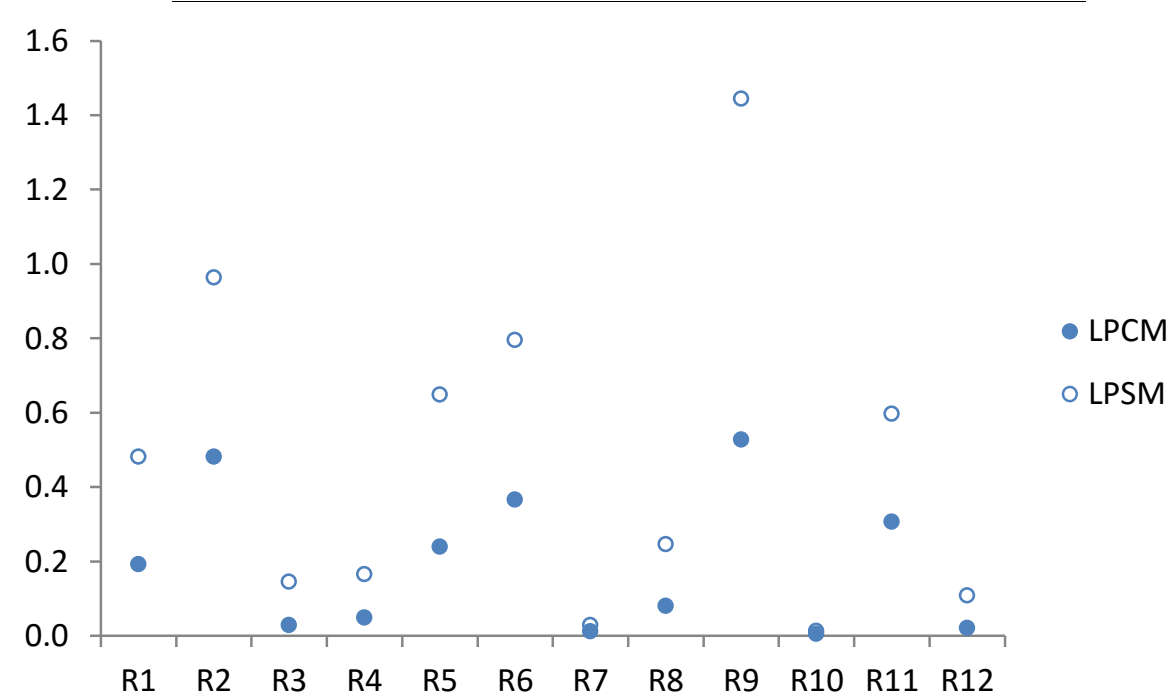

Fig. 3. Risk spectrum of the supply network under standard configuration

Network expected loss is an important parameter that reflects the risk level of the supply network under given conditions. Variation of network expected loss with all combinations of control strategies is shown in Fig. 4. Generally, network expected loss decreases with the increase in mitigation cost. However, corresponding to different cost regimes, it might not be viable to implement costly strategies because of the interdependent nature of these strategies with risks across the network. For each cost value, the optimal combination of strategies is represented by a solid circle whereas 
hollow circles represent inefficient solutions. This model helps in identifying inefficient solutions as well.

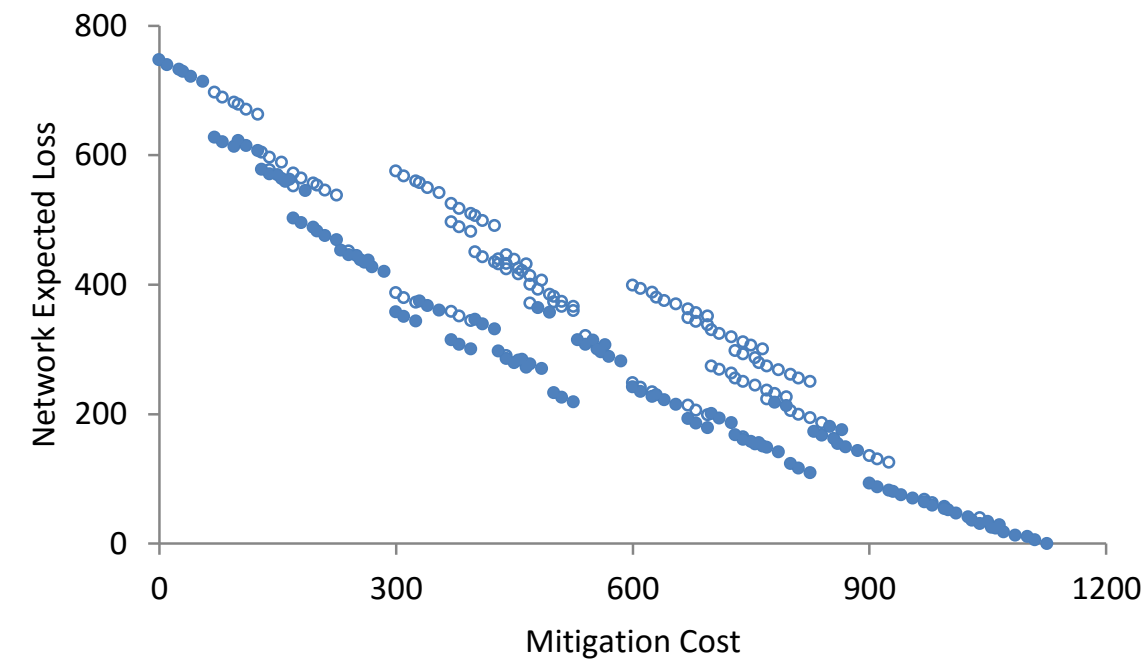

Fig. 4. Variation of network expected loss with different combinations of risk mitigation strategies and associated cost

Average LPCM is a measure of relative percentage improvement in the network expected loss with the overall average improvement in the state of each risk factor. Average LPCM decreases with the increase in mitigation cost as shown in Fig. 5. It means that because of the implementation of control strategies, the risk condition of individual risk factors improves and therefore, the relative margin of improvement for the network expected loss is reduced. Each combination of control strategies representing minimum value of average LPCM corresponding to specific mitigation cost is shown in solid circle. Implementing preventive strategies at all control nodes of the network results in achieving average LPCM of 0 at the cost of 1125 units. It is also interesting to observe wide variation of optimal points with the increase in mitigation cost.

Average LPSM is a measure of relative percentage variation in the network expected loss with the overall average variation in the state of each risk factor. In general, average LPSM also decreases with the increase in mitigation cost as shown in Fig. 6. In case of implementing reactive strategies, LPSM decreases as the resulting loss is reduced, however, choice of a preventive strategy reduces the probability of risk event without affecting the value of resulting loss and therefore, LPSM is not reduced. It can be observed that average LPSM starts increasing after a certain value of mitigation cost (approx. 640 units) because of incorporating preventive strategies in the portfolio of mitigation strategies. Each combination of control strategies representing minimum value of average LPSM corresponding to specific mitigation cost is shown in solid circle. Similar to the case of average LPCM, it is also interesting to observe wide variation of optimal points with the increase in mitigation cost. 


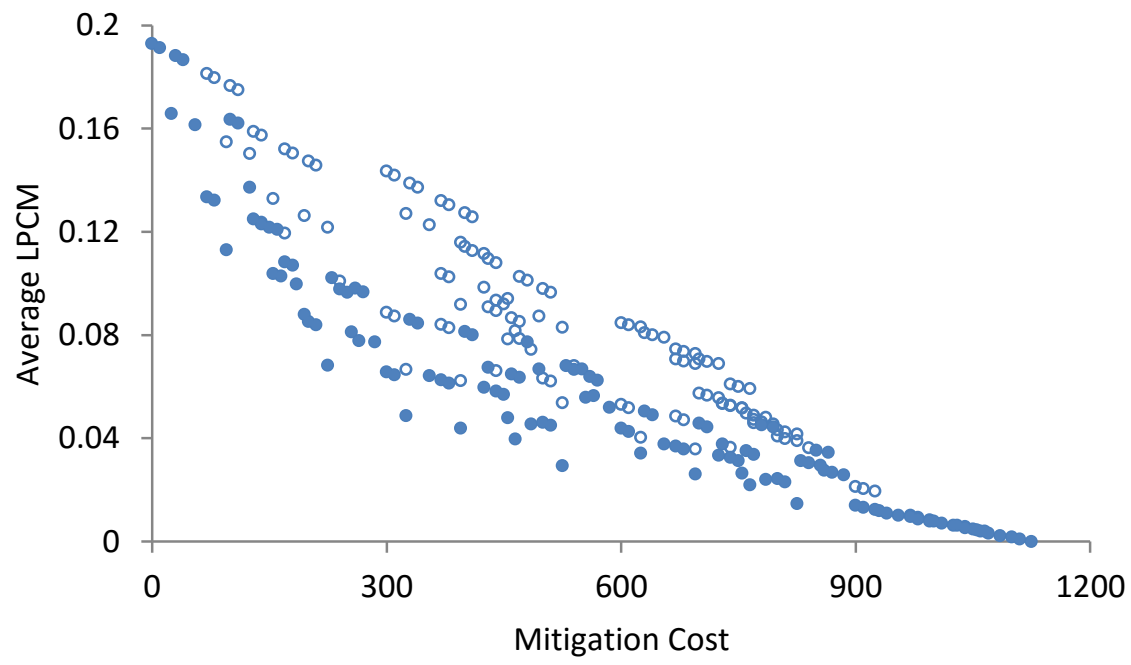

Fig. 5. Variation of average LPCM with different combinations of control strategies and associated cost

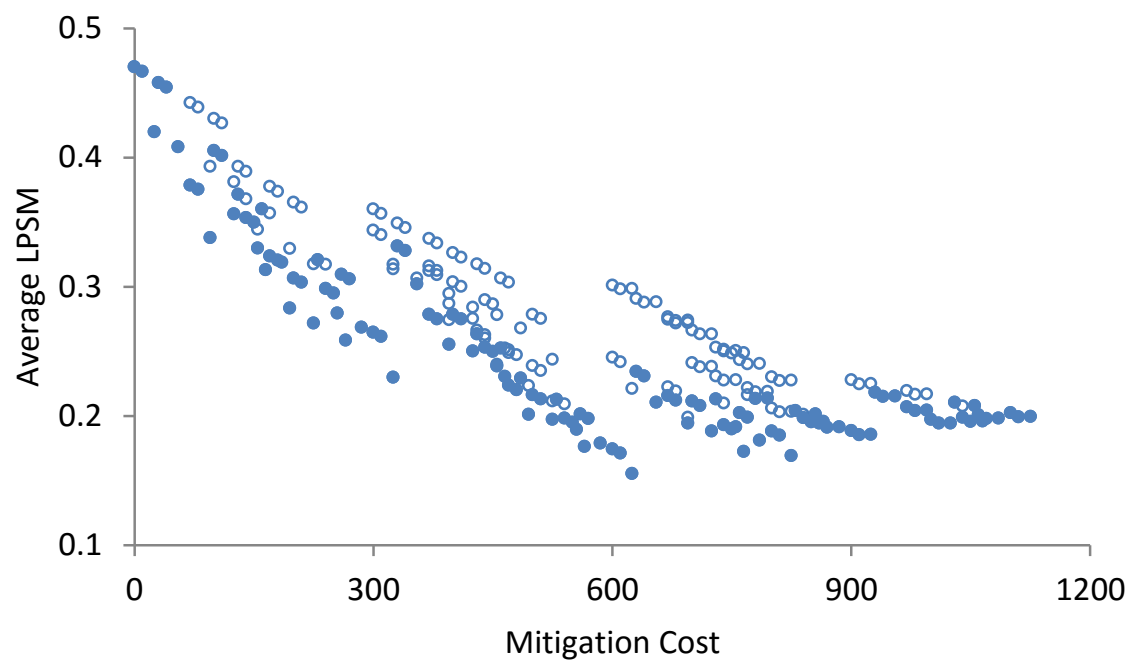

Fig. 6. Variation of average LPSM with different combinations of control strategies and associated cost

As our objective function necessitated selection of an optimal combination of strategies resulting in the maximum value of relative improvement of network expected loss taking into account the associated cost of mitigation strategies, it is important to consider the variation of this function with different combinations of control strategies as shown in Fig. 7. The graph reveals that the maximum value of objective function is achieved at the mitigation cost of 300 units. However, there are two 
other inefficient solutions and without the help of this modelling technique, the relative impact of each specific combination of strategies might not be appreciated. Combinations of optimal and inefficient strategies are presented in Table 4. It is interesting to find that one of the inefficient solutions requires implementing a strategy at the most important risk factor R9, however, keeping in view the interdependency between different factors, such a solution is not viable. Furthermore, it is important to consider that a decision maker might not treat the expected loss and mitigation cost equally in evaluating the optimal choice of strategies. Expected loss may be assigned more weightage keeping in view the reputational risks and other non-monetary factors.

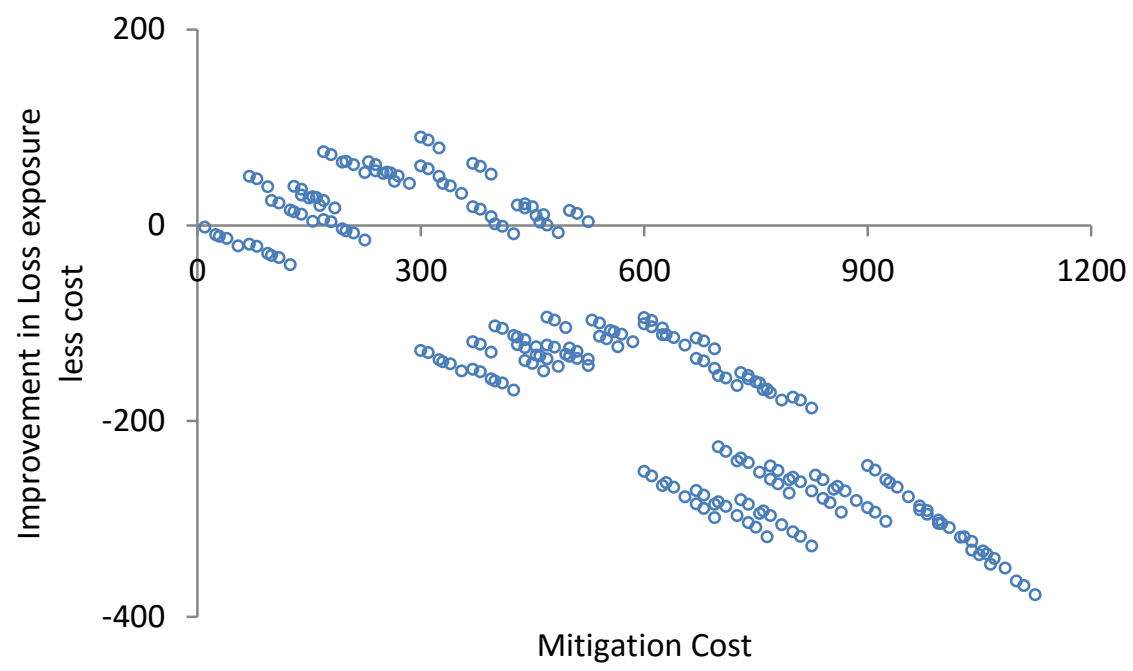

Fig. 7. Cost and benefit analysis of various mitigation strategies

Table 4. Combinations of optimal and inefficient strategies

\begin{tabular}{cccc}
\hline \multirow{2}{*}{ Risk } & \multicolumn{3}{c}{ Portfolio of Risk Mitigation Strategies } \\
\cline { 2 - 4 } & Optimal & Inefficient 1 & Inefficient 2 \\
\hline R2 & Reactive & No & Preventive \\
R5 & Preventive & No & No \\
R6 & Preventive & No & No \\
R9 & No & Reactive & No \\
R11 & No & No & No \\
\hline$E L(X)$ & 357.62 & 575.56 & 387.26 \\
\hline \multicolumn{5}{c}{ Total Cost } & \multicolumn{3}{c}{300} \\
\hline
\end{tabular}

Risk measures of all risk factors under contingency configuration are shown in Table 5 and risk spectrum representing the graphical dimension of risk measures is shown in Fig. 8. As preventive strategies were implemented at R5 and R6, their LPCM values are 0. LPCM and LPSM values for R3 and R4 are all 0 because their impact is blocked by their descendant nodes. Although no mitigation strategy is implemented at R9, its LPCM and LPSM values have decreased because of the impact 
of strategies implemented at R5 and R6. R9 still remains a critical risk factor, however, keeping in view the relative costs of implementing strategies, it is not viable to protect this node.

Table 5. Risk measures of risk factors under contingency configuration

\begin{tabular}{ccccc}
\hline & \multicolumn{4}{c}{ Contingency Configuration } \\
\cline { 2 - 5 } Risk & $\begin{array}{c}\text { Expected } \\
\text { Loss (True) }\end{array}$ & $\begin{array}{c}\text { Expected } \\
\text { Loss (False) }\end{array}$ & LPCM & LPSM \\
\hline R1 & 432.62 & 307.62 & 0.0669 & 0.1672 \\
R2 & 482.62 & 232.62 & 0.1672 & 0.3344 \\
R3 & 357.62 & 357.62 & 0.0000 & 0.0000 \\
R4 & 357.62 & 357.62 & 0.0000 & 0.0000 \\
R5 & 615.36 & 357.60 & 0.0000 & 0.3448 \\
R6 & 672.86 & 357.59 & 0.0000 & 0.4218 \\
R7 & 364.48 & 353.05 & 0.0061 & 0.0153 \\
R8 & 421.65 & 326.37 & 0.0418 & 0.1275 \\
R9 & 1088.45 & 135.83 & 0.2967 & 1.2744 \\
R10 & 363.98 & 353.38 & 0.0057 & 0.0142 \\
R11 & 527.93 & 213.83 & 0.1924 & 0.4202 \\
R12 & 391.16 & 349.24 & 0.0112 & 0.0561 \\
\hline \multicolumn{5}{c}{} \\
\hline
\end{tabular}

Evaluation of risk mitigation strategies through our proposed approach results in an optimal mix of preventive and reactive strategies. As our approach incorporates interdependency between supply network elements, risks and mitigation strategies and follows rigorous technique of BBNs, the resulting solution can be considered as viable. However, it is assumed that all the stakeholders would be willing to share their private information and furthermore, elicited values would truly reflect the real-time risk scenario. Besides the limitations associated with modelling huge supply networks, these assumptions are deemed as challenges to our proposed approach.

\subsection{Managerial Implications}

The proposed modelling approach can help supply chain managers visualize interdependency between supply chain risks across the supply network. Stakeholders can identify important triggers and risk events and evaluate the impact of different risk mitigation strategies on the entire web of interconnected risks. Furthermore, if stakeholders consider only their domain of the supply network, they might implement strategies yielding sub-optimal solutions and therefore, it is extremely important to involve all stakeholders in this modelling process for achieving the global optimal solution. Causal mapping (qualitative modelling of BBNs) is beneficial to the managers in identifying important risks and understanding the dynamics between these risks. It is also important to realize that crucial decision of selecting an optimal mix of pre- 
ventive and reactive strategies can only be made after following the proposed rigorous approach of modelling interdependency between risks and mitigation strategies.

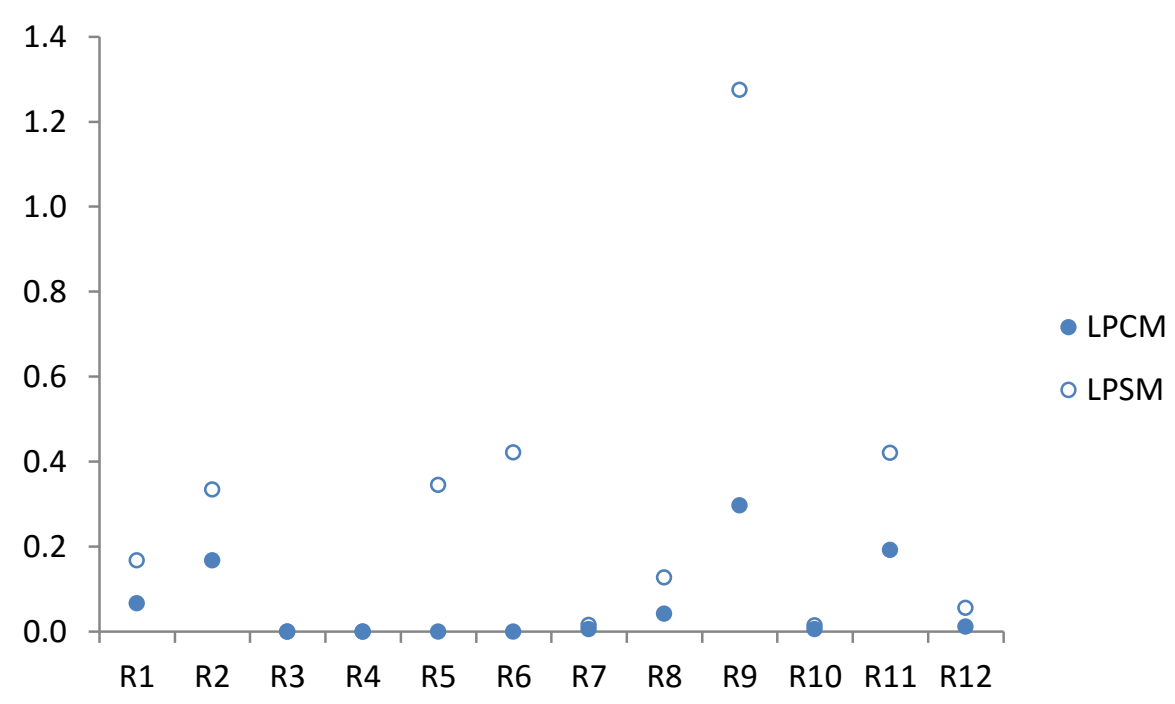

Fig. 8. Risk spectrum of the supply network under contingency configuration

\section{$5 \quad$ Conclusion and Future Research}

Generally, available models in the field of Supply chain risk management address specific problems, whereas, few models capturing interdependency between risks do not cover all stages of risk management process. We have bridged this important research gap and proposed a new approach of modelling interdependency between risks and evaluating different control strategies (preventive and reactive). Optimal combination of strategies can only be selected after adopting a rigorous modelling approach of capturing supply network configuration, probabilistic interdependency between risks, resulting losses and costs associated with different risk mitigation strategies. Our proposed risk measures are easy to compute and reflect the contribution of risk factors towards the network expected loss. We have also demonstrated use of our proposed method through a simple simulation study.

Our model is based on a number of assumptions. Firstly, the method may be feasible for a limited network and in case of a large network, elicitation of (conditional) probability values may be cumbersome. However, this problem can be tackled with introducing some assumptions in the model itself like Noisy-OR function. Secondly, we assume that stakeholders would be willing to share true information of the risks and loss values, however, it might not be in the best interest of stakeholders to share private information and therefore, they would need to be incentivized for developing the model and sharing real data.

We have also assumed binary states for all the risk factors. However, future research may focus on representing risks by continuous variables. Furthermore, a con- 
trol strategy may also be represented by a continuum of control levels and associated costs. Our proposed method can help researchers develop robust models for managing supply chain risks. Supply chain managers can visualize the interaction between different risks and appreciate the importance of key risk factors. In future, the proposed method may be applied in modelling real supply networks in order to evaluate its efficacy.

\section{References}

1. Jüttner, U., Peck, H., and Christopher, M., Supply chain risk management: outlining an agenda for future research. International Journal of Logistics Research and Applications, 2003. 6(4): pp. 197-210.

2. Christopher, M. and Peck, H., Building the Resilient Supply Chain. International Journal of Logistics Management, 2004. 15(2): pp. 1-14.

3. Son, J.Y. and Orchard, R.K., Effectiveness of policies for mitigating supply disruptions. International Journal of Physical Distribution and Logistics Management, 2013. 43(8): pp. 684-706.

4. Badurdeen, F., Shuaib, M., Wijekoon, K., Brown, A., et al., Quantitative modeling and analysis of supply chain risks using Bayesian theory. Journal of Manufacturing Technology Management, 2014. 25(5): pp. 631-654.

5. Qazi, A., Quigley, J., and Dickson, A. A novel framework for quantification of supply chain risks. in 4th Student Conference on Operational Research. 2014. University of Nottingham, UK.

6. Khan, O. and Burnes, B., Risk and supply chain management: creating a research agenda. International Journal of Logistics Management, 2007. 18(2): pp. 197-216.

7. White, D., Application of systems thinking to risk management: a review of the literature. Management Decision, 1995. 33(10): pp. 35-45.

8. Tang, C.S., Perspectives in supply chain risk management. International Journal of Production Economics, 2006. 103(2): pp. 451-488.

9. Qazi, A., Quigley, J., and Dickson, A. Supply Chain Risk Management: Systematic literature review and a conceptual framework for capturing interdependencies between risks. in 5th International Conference on Industrial Engineering and Operations Management. 2015. Dubai, UAE.

10. Gaudenzi, B. and Borghesi, A., Managing risks in the supply chain using the AHP method. International Journal of Logistics Management, 2006. 17(1): pp. 114-136.

11. Garvey, M.D., Carnovale, S., and Yeniyurt, S., An analytical framework for supply network risk propagation: A Bayesian network approach. European Journal of Operational Research, 2015. 243(2): pp. 618-627.

12. Lockamy, A. and McCormack, K., Analysing risks in supply networks to facilitate outsourcing decisions. International Journal of Production Research, 2009. 48(2): pp. 593611.

13. Dogan, I. and Aydin, N., Combining Bayesian Networks and Total Cost of Ownership method for supplier selection analysis. Computers and Industrial Engineering, 2011. 61(4): pp. 1072-1085.

14. Sodhi, M.S. and Tang, C.S., Managing supply chain risk, International Series in Operations Research and Mangement Science 172. Vol. 172. 2012: New York : Springer.

15. Wagner, S.M. and Bode, C., An empirical investigation into supply chain vulnerability. Journal of Purchasing and Supply Management, 2006. 12(6): pp. 301-312. 